\title{
Narrative review of intraoperative image guidance for transforaminal lumbar interbody fusion
}

\author{
Joseph A. Weiner ${ }^{1}$, Michael H. McCarthy ${ }^{2}$, Peter Swiatek ${ }^{1}$, Philip K. Louie' ${ }^{2}$, Sheeraz A. Qureshi ${ }^{2}$ \\ ${ }^{1}$ Northwestern University Department of Orthopaedic Surgery, Chicago, IL, USA; ${ }^{2}$ Hospital for Special Surgery Department of Orthopedic Surgery, \\ New York, NY, USA \\ Contributions: (I) Conception and design: JA Weiner, MH McCarthy, PK Louie, SA Qureshi; (II) Administrative support: None; (III) Provision of \\ study materials or patients: None; (IV) Collection and assembly of data: JA Weiner, PR Swiatek; (V) Data analysis and interpretation: All authors; (VI) \\ Manuscript writing: All authors; (VII) Final approval of manuscript: All authors. \\ Correspondence to: Joseph A. Weiner, MD. Department of Orthopaedic Surgery, 676 N St Clair, 13th floor, Chicago, IL 60611, USA. \\ Email: Joseph.weiner@northwestern.edu.
}

\begin{abstract}
Recent advancements in imaging technology have changed the landscape of transforaminal lumbar interbody fusion (TLIF) with the objective of improving safety and efficacy for the patient and surgical team. Spine surgery, and specifically TLIFs, involve challenging anatomy and command precise surgical accuracy, creating an essential role for intraoperative imaging, navigation, and robotics. Traditionally, surgeons have relied upon fluoroscopy for pedicle screw and interbody placement. More recently, intraoperative 3-dimensional navigation (ION) has risen in popularity in TLIF surgery. This technology utilizes intra-operative advanced imaging, such as computed tomography (CT) and 3D-fluroscopy, to accurately track instruments and implants in relation to the patient's anatomy. ION has demonstrated improved accuracy of pedicle screw placement, decreased operating room times, and lower radiation exposure to the surgeon and staff. However, conventional fluoroscopy, 3D fluoroscopy, intraoperative CT, image-guided navigation, and robot-assisted surgery all have a role in TLIF surgery. Numerous studies have been published regarding the benefits and pitfalls of these intraoperative tools in spine surgery, but there is a relative lack of research regarding some of the newer technologies surrounding TLIF. As future studies are published, and technology continues to evolve, surgeons must stay abreast of novel techniques to maximize patient safety and outcomes. Over the coming decade, we can expect intraoperative navigation and robotics to play a more significant role in spine surgery.
\end{abstract}

Keywords: Intraoperative imaging; transforaminal lumbar interbody fusion (TLIF); spine surgery; computed tomography navigation (CT navigation); robotic spine surgery

Submitted Feb 26, 2020. Accepted for publication Jul 01, 2020.

doi: 10.21037/atm-20-1971

View this article at: http://dx.doi.org/10.21037/atm-20-1971

\section{Introduction}

In the past several decades, fusion for degenerative spine disorders has become one of the most commonly performed surgical procedures (1). After the introduction of interbody cages and FDA approval of pedicle screws in the mid1990s, rates of spine fusion from 1990 to 2001 increased by $220 \%$ (2). More recent data have shown continued growth, with a $137 \%$ increase in the rate of lumbar fusion from
1998 to 2008, significantly outpacing the growth in total hip and knee replacement during the same period (3).

Interbody fusion techniques have gained popularity for the treatment of various degenerative spine conditions because of the asserted benefit of providing direct anterior column support, circumferential fusion, indirect neural foraminal decompression, and restoration of lumbar lordosis $(4,5)$. The utilization of interbody fusion for degenerative spondylolisthesis increased significantly, from $13.6 \%$ in 
1999 to $32 \%$ in 2011 (4). Advances in surgical techniques and novel imaging technologies have facilitated the development of less morbid interbody fusion procedures, such as transforaminal lumbar interbody fusion (TLIF).

Harms and Rolinger introduced TLIF in 1982. The technique involved a unilateral approach to the disc space through the ipsilateral facet joint, enabling placement of an interbody spacer to achieve indirect decompression of affected nerve roots (6). Further advances in TLIF over the past several decades focused on reducing morbidity through the development of minimally invasive techniques (MIS-TLIF); intraoperative imaging modalities and tubular retractor systems enabled these techniques (7). MIS-TLIF results in less blood loss and shorter hospital stay but requires increased reliance on intraoperative imaging with longer $\mathrm{X}$-ray exposure time compared with open TLIF technique (8). In this review of intraoperative image guidance for TLIF we will review the techniques, benefits, and pitfalls of: (I) conventional fluoroscopy; (II) 3D fluoroscopy; (III) intraoperative CT; (IV) navigation; (V) robotic spine surgery. We present the following article in accordance with the Narrative Review reporting checklist (available at http://dx.doi.org/10.21037/atm-20-1971).

\section{Methods}

This study identified literature including randomized controlled trials (RCT), observational case-control studies, prospective and retrospective cohort studies, technique guides, and reviews reporting on intraoperative imaging in TLIF intraoperative navigation during TLIF, and robotassisted TLIF. The National Institutes of Health PubMed database was queried using a combination of free and medical subject headings search parameters related to the surgical intervention. Two authors independently conducted a systematic PubMed search to identify studies published between January 1, 1980 and December 31, 2019. This search was performed using combinations of the following search terms: "Transforaminal lumbar interbody fusion" "TLIF" "intraoperative imaging" "intraoperative navigation" "navigated TLIF" "robotic TLIF". All English language studies on human subjects were included. The cited papers in the identified articles were also systematically reviewed, ensuring that all eligible items were identified and included.

\section{Conventional fluoroscopy}

During MIS TLIF, appropriate anatomic landmarks for freehand insertion of pedicle screws and interbody implants are not exposed. Thus, the MIS approach comes with an increased reliance on intraoperative imaging for confirming anatomic levels, guiding pedicle screw placement, and determining local spinal alignment. This image guidance is most commonly performed with conventional C-arm fluoroscopy, such as GE OEC Elite (GE Healthcare, Chicago, IL) (9).

\section{Technique}

During MIS-TLIF using conventional fluoroscopy, the patient is positioned prone on a radiolucent table. Fluoroscopy is classically involved in 5 steps during MISTLIF: preoperative incisional skin marking, retractor positioning, placement of the interbody cage, insertion of the percutaneous pedicle screws, and placement of the rods. Initial anteroposterior (AP) images are obtained to verify proper patient positioning with the spinous process midline between the pedicles of each level. Based on surgeon preference, initial AP images can be utilized to mark out the location of the desired pedicles and disc spaces. The C-arm is then moved into the lateral position and the correct levels are verified.

After initial surgical exposure utilizing the Wiltse approach, a tubular retractor can be placed under lateral fluoroscopic guidance. The retractor is docked over the ipsilateral facet joint of the selected level using a rigid arm attached to the operating table, and successive dilators are placed until a retractor of sufficient diameter is reached. This allows for the facetectomy and discectomy to occur under direct visualization with the assistance of loupes or operating microscope.

After standard preparation of the disc space, interbody spacer trials can be inserted to determine the proper implant size. Lateral fluoroscopic views can be utilized to assess the restoration of local lumbar lordosis, and AP views can be used to determine the proper medial/lateral positioning of the implant. Finally, the implant of choice can be placed with lateral and AP fluoroscopic views utilized to verify the positioning of the interbody cage implant.

The most critical role for fluoroscopy during MIS-TLIF is for percutaneous pedicle screw placement. Minimally invasive percutaneous pedicle screw placement was first performed in the late 1970s during external fixation of the spine in the setting of trauma $(10,11)$. The early success of pedicle based fixation led to the development of internal fixation systems for segmental lumbar fusion 
using pedicle screws $(7,12-15)$. In this technique, a small paramedian incision is created over the selected pedicle, and a transpedicular passage is created using the Jamshidi needle under AP and lateral fluoroscopic guidance into the upper outer quadrant of the pedicle. The needle is advanced approximately $30 \mathrm{~mm}$ while verifying that it does not cross the medial border of the pedicle. Guidewires are subsequently inserted into the pedicles through the needles. Insertion is confirmed on AP and lateral imaging. Serial dilation then occurs over the guidewire. The pedicles are tapped with a cannulated tap. Finally, the dilators are removed and the cannulated screws are introduced over the guide wires. All screws are confirmed with AP and lateral fluoroscopy.

\section{Pitfalls}

Although MIS-TLIF is advantageous for patients with lumbar degenerative diseases, minimally invasive procedures performed under fluoroscopy have a few disadvantages that have led to the development of enhanced imaging and navigation technologies. Fluoroscopic techniques have resulted in pedicle screw malposition rates reported between $1.5 \%$ and $13 \%$ (16). The 2-dimensional nature of traditional $\mathrm{C}$-arm fluoroscopy makes the accurate placement of pedicle screws challenging for inexperienced providers. Another common disadvantage often cited is that reliance on fluoroscopy has increased radiation exposure to surgeons and operating room staff (9). Several studies have demonstrated that conventional C-arm fluoroscopy results in higher radiation doses for surgeons and operating room staff $(17,18)$. In 2012, Tabaraee et al. reported that the average exposure to a surgeon's thyroid during MIS spine surgery utilizing conventional fluoroscopy was $0.1 \mathrm{mSv}$. The International Commission on Radiological Protection (ICRP) recommends a dose limit of $20 \mathrm{mSv}$ averaged over five years with no single year having more than $50 \mathrm{mSv}$ exposure. Based on the ICRP recommended yearly limit of $20 \mathrm{mSv}$ per year, surgeons would only be able to complete 200 procedures per year safely (19). A recent meta-analysis revealed that MIS-TLIF resulted in double the radiation exposure for the patient and operating room staff compared to open TLIF (20).

\section{D fuoroscopy}

First introduced in 2001, 3D fluoroscopy utilizes a series of $2 \mathrm{D}$ images to reconstruct a $3 \mathrm{D}$ intraoperative image.
The Siremobil Iso-C 3D (Siemens, Munich, Germany) was the first mobile $\mathrm{C}$-arm to provide an intraoperative 3D image (21). 3D fluoroscopy uses an automatic orbital rotation around the patient to obtain a series of successive 2D fluoroscopic images. Using a computer algorithm, the images are used to generate a $3 \mathrm{D}$ reconstruction that is displayed as axial, sagittal, and coronal planes. The resulting images are similar to what is typically obtained with a CT scan.

Over the last decade, the growth of $3 \mathrm{D}$-fluoroscopy has resulted in a significant reduction in the misplacement rate of pedicle screws $(22,23)$. The principal advantage provided by modern $\mathrm{C}$-arm devices is the capability of providing conventional two-dimensional (2D) fluoroscopic images, as well as 3D images for intraoperative navigation. During TLIF procedures, 3D C-arms can interface with intraoperative navigation systems to guide pedicle screw and interbody placement. The utility of intraoperative navigation will be discussed in detail in a later section.

\section{Intraoperative CT}

In May 2005, the O-arm imaging system (Medtronic, Minneapolis, MN) was approved by the Food and Drug Administration for intraoperative use based on equivalency to 3D fluoroscopy systems (24). 2D image guidance systems only offer navigation in coronal and sagittal planes; however, clinically significant pedicle violations are most often detected on axial imaging. Similar to 3D fluoroscopy, the principal advantage of intraoperative CT comes from the ability to interface with navigation systems. However, the scanned volume is three times larger than that obtained from the 3D C-arm (17). Thus, the O-arm system scan can capture multiple levels in one scan, reducing the need for changing the field of interest and allowing the surgeon to execute cases where large areas need to be visualized.

During TLIFs, the patient is positioned supine on a radiolucent table. After the patient is prepped and draped in a sterile fashion, a static reference frame is placed percutaneously into the posterior superior iliac spine. The navigation system uses this array to determine the location of instruments in space relative to the static anatomy. The intraoperative CT scanner is sterilely draped then brought in and a scan of the operative levels is obtained. This scan is then used by the intraoperative navigation system to provide real-time axial, coronal, and sagittal images.

One key advantage of intraoperative CT is the decrease in the surgeon and staff radiation exposure (25-29). During 
the initial scan, the surgeon and operating room staff can exit the room, thereby limiting their exposure. Although the dose of radiation delivered to the patient intraoperatively may be nearly double with CT compared to fluoroscopy, average total radiation exposure to the patient is still around $5.6 \mathrm{mSv}$, a value less than a single routine lumbar CT scan (7.5 mSv) (27).

\section{Navigation}

With both 3D fluoroscopy and intraoperative CT, the fundamental advantage stems from the use of navigation. Intraoperative image-based navigation was initially adapted from intracranial neurosurgery in the early 1990s, where frameless stereotaxy was utilized for navigation within the delicate intracranial anatomy (30). The initial application for cranial surgery used skin surface markers for registration, thereby allowing the computer to match the patient anatomy to the preoperative imaging. Unfortunately, these skin markers were not useful in spine surgery due to relative movement between the skin surface and the underlying bony anatomy (31).

Despite the initial difficulties with navigation, the issue of registration was alleviated by utilizing a dynamic reference array that is fixed to the patient's bony anatomy before obtaining the CT scan (32). A typical modern navigation system is composed of a dynamic reference array, an optical camera array, and customized surgical instruments (awls, probes, etc.) that contain reflective spheres. These reflective spheres, attached to the reference array and surgical instruments, are tracked by the optical camera array. A computer workstation running proprietary software measures the three-dimensional location of the arrays in the operating room. The spatial location of the instruments is displayed in relation to the intraoperative imaging on the computer monitor. There are numerous commercially available navigation systems FDA approved for use during TLIF, including Stealth (Medtronic), Brainlab, and Navi3i (Stryker).

The advantages of intraoperative navigation have been extensively reported $(8,19,24,26,27,32-35)$. The main advantages of intraoperative navigation in spine surgery are: increased accuracy of pedicle screw placement $v$ s. freehand techniques, improved ability to use minimally invasive techniques, and decreased radiation exposure to the surgeon and operating room staff. The most studied advantage of navigation utilization during TLIF is the increased accuracy of pedicle screw placement $(24,36)$. Utilizing the real-time feedback provided by navigation, accurate start points and trajectories can be identified without exposing the vertebral anatomy. Furthermore, the actual position of the screw can be monitored in real-time to avoid critical structures, including neural elements. Numerous metaanalyses have identified the breach rate for non-navigated pedicle screws in the thoracolumbar spine to be anywhere between $15-20 \%(24,36)$. In a separate meta-analysis of pedicle screw accuracy using traditional fluoroscopy, the breach rate improved to $13 \%$ (8). Individual cases series of non-navigated and traditional fluoroscopic techniques have pedicle screw breach rates as low as $2-6 \%$, suggesting significant variability between individual surgeons $(37,38)$.

Large multicenter meta-analyses have demonstrated the pedicle screw misplacement rate for intraoperative navigation to be between $2-6 \%(23,36)$. Smaller case series have reported these rates as low as $1-2 \%$ with the use of advanced intraoperative navigation $(39,40)$. Although most studies have included pedicle screws placed in various types of posterior spinal fusions, a recent 2018 study by Dusad et al. analyzed the use of navigation in MIS-TLIF. They reported $96.29 \%(104 / 108)$ of pedicle screws in the navigation group were accurately placed compared with 91.67\% (220/240) in the non-navigation group; however, the difference was not statistically significant (41).

Another purposed benefit of navigation is the ability to optimize pedicle screw biomechanics. It has been demonstrated that pedicle screw medialization, greater screw outer diameter, and greater cortical engagement contribute to improved screw pullout strength. Furthermore, increased screw inner diameter improves fatigue strength (42). Image-guided navigation allows the surgeon to perfect screw placement and maximize these variables intraoperatively.

As previously discussed, intraoperative CT has the added benefit of lower radiation exposure for the surgeon and operating room staff. This benefit stems from the ability to assess screw and cage placement without additional intraoperative imaging. Thus, the reduction in radiation exposure carries over to all navigation-based imaging techniques $(43,44)$. Scheufler et al. reported on 30 patients with adult degenerative scoliosis treated by minimally invasive decompression and unilateral TLIF at the convexity of the deformity using biplanar fluoroscopy or intraoperative CT-based navigation (45). Single-level TLIFs utilizing fluoroscopic guidance resulted in surgeon radiation exposure of $0.025 \mathrm{mSv}$. In contrast, radiation exposure was $0 \mathrm{mSv}$ with intraoperative CT navigated instrumentation. Notably, navigation provided a significantly faster time 
to complete pedicle screw insertion than guidance with biplanar fluoroscopy.

\section{Robotics}

Robot-assisted spine surgery has blossomed over the past decade as a practical tool to advance the field of minimally invasive spine surgery. The first-ever spine robot, the SpineAssist (Mazor Robotics Ltd., Caesarea, Israel), gained FDA approval as a tool for pedicle screw placement in 2004. Since then, newer robotic devices including the Mazor X (Medtronic and Mazor Robotics, Memphis, TN, USA), ExcelsiusGPS (Globus Medical, Inc., Audubon, PA, USA), and ROSA (Medtech Surgical, Inc., New York, NY, USA) have been developed, and FDA cleared for use in spine surgery.

All of the FDA approved robots are capable of providing real-time intraoperative navigation and rigid stereotaxy for inserting pedicle screws. They are shared-control systems that allow for the surgeon and robot to directly control the surgical instruments simultaneously (46). They utilize a similar workflow to navigated freehand pedicle screw insertion. Utilizing intraoperative imaging, surgeons can plan stereotactic trajectories, which are, in turn, produced by an automated robotic arm (47).

Compared to convention fluoroscopic guided surgery, robotic-assisted surgery has demonstrated increased accuracy while decreasing radiation exposure, complication rates, operative time, and recovery time $(48,49)$. While there is a lack of evidence comparing robot-assisted to surgery to intraoperative navigation alone, the robot does offer several other benefits, including collision avoidance, eliminating hand tremors, reducing surgeon fatigue, decreasing incision size, and providing more degrees of freedom than the human extremity (50). Lastly, Kim et al. recently reported that robot-assisted pedicle screw placement is less likely to damage adjacent facet joints, likely leading to lower rates of adjacent segment disease (51). This is theorized to be due to more optimized entry points away from the adjacent facet and the lack of accidental penetration of the adjacent facet capsule when attempting to find the correct start point using the freehand technique.

There is an overall paucity of literature on outcomes of robotic-assisted TLIF. During TLIF surgery, the primary role of robot assistance is percutaneous pedicle screw placement. Decompression, preparation of the disc space, and insertion of the interbody device are all performed using standard technique under image navigation guidance (52).
Chenin et al. published a report of their technical integration and surgical experiences utilizing ROSA (Medtech Surgical, Inc., New York, NY, USA) spine robot and intraoperative $\mathrm{O}$-arm CT navigation for minimally invasive TLIFs (53).

In 2017 , Du et al. published the results of 96 robotassisted minimally invasive TLIFs for degenerative scoliosis. The primary goal of their study was to analyze the impact of placing a gelatin-coated sponge soaked with a mixture of steroid, anesthetic, and vitamin B12. They used the MAZOR robot to place 922 pedicle screws in 96 patients and reported a $>1 \mathrm{~mm}$ breach rate of $1.3 \%$ with no resulting neurologic complications (54).

Overall, the massive growth of robot-assisted spine surgery over the past decade has been mainly focused on pedicle screw instrumentation. The current literature indicates that robot-assisted pedicle screw placement during TLIF is a safe and effective technique that likely results in improved accuracy with reduced radiation exposure for the surgical team. Future directions for robotics likely include robot-assisted decompression and facetectomies.

\section{Conclusions}

Advancements in intraoperative imaging technology and MIS spine surgery have resulted in the evolution of TLIF surgery. Traditional fluoroscopic guidance for TLIF procedures is slowly being replaced by advanced image-guided navigation utilizing $3 \mathrm{D}$ fluoroscopy and intraoperative CT. The majority of current literature supports increased accuracy during pedicle screw instrumentation, decreased procedure time, and decreased radiation exposure to the surgeon and operating room staff with image-guided navigation. New frontiers in TLIF surgery include continued development of robotic assisted surgery for both implant and pedicle screw placement.

\section{Acknowledgments}

Funding: None.

\section{Footnote}

Provenance and Peer Review: This article was commissioned by the Guest Editor (Dr. Sheeraz A. Qureshi) for the series "Current State of Intraoperative Imaging" published in Annals of Translational Medicine. The article was sent for external peer review organized by the Guest Editor and the 
editorial office.

Reporting Checklist: The authors have completed the Narrative Review reporting checklist. Available at http:// dx.doi.org/10.21037/atm-20-1971

Conflicts of Interest: All authors have completed the ICMJE uniform disclosure form (available at http://dx.doi. org/10.21037/atm-20-1971). The series "Current State of Intraoperative Imaging" was commissioned by the editorial office without any funding or sponsorship. SAQ served as the unpaid Guest Editor of the series and serves as an unpaid editorial board member of Annals of Translational Medicine from Sep 2019 to Aug 2021. SAQ reports personal fees from Globus Medical, Inc., personal fees and other from Healthgrades, other from Avaz Surgical, personal fees from Paradigm Spine, personal fees from Stryker, other from Vital 5, outside the submitted work. The authors have no other conflicts of interest to declare.

Ethical Statement: The authors are accountable for all aspects of the work in ensuring that questions related to the accuracy or integrity of any part of the work are appropriately investigated and resolved.

Open Access Statement: This is an Open Access article distributed in accordance with the Creative Commons Attribution-NonCommercial-NoDerivs 4.0 International License (CC BY-NC-ND 4.0), which permits the noncommercial replication and distribution of the article with the strict proviso that no changes or edits are made and the original work is properly cited (including links to both the formal publication through the relevant DOI and the license). See: https://creativecommons.org/licenses/by-nc-nd/4.0/.

\section{References}

1. Makanji H, Schoenfeld AJ, Bhalla A, et al. Critical analysis of trends in lumbar fusion for degenerative disorders revisited: influence of technique on fusion rate and clinical outcomes. Eur Spine J 2018;27:1868-76.

2. Deyo RA, Gray DT, Kreuter W, et al. United States trends in lumbar fusion surgery for degenerative conditions. Spine (Phila Pa 1976) 2005;30:1441-5.

3. Rajaee SS, Bae HW, Kanim LEA, et al. Spinal fusion in the United States: Analysis of trends from 1998 to 2008. Spine (Phila Pa 1976) 2012;37:67-76.

4. Kepler CK, Vaccaro AR, Hilibrand AS, et al. National trends in the use of fusion techniques to treat degenerative spondylolisthesis. Spine (Phila Pa 1976) 2014;39:1584-9.

5. Sudo H, Oda I, Abumi K, et al. Biomechanical study on the effect of five different lumbar reconstruction techniques on adjacent-level intradiscal pressure and lamina strain. J Neurosurg Spine 2006;5:150-5.

6. Harms J, Rolinger H. A One-Stager Procedure in Operative Treatment of Spondylolistheses: Dorsal Traction-Reposition and Anterior Fusion (Author's Transl). Z Orthop Ihre Grenzgeb 1982;120:343-7.

7. Foley KT, Holly LT, Schwender JD. Minimally invasive lumbar fusion. Spine (Phila Pa 1976) 2003;28:S26-35.

8. Tian NF, Xu HZ. Image-guided pedicle screw insertion accuracy: a meta-analysis. Int Orthop 2009;33:895-903.

9. Wang TY, Farber SH, Perkins SS, et al. Internally Randomized Control Trial of Radiation Exposure Using Ultra-low Radiation Imaging Versus Traditional C-arm Fluoroscopy for Patients Undergoing Single-level Minimally Invasive Transforaminal Lumbar Interbody Fusion. Spine (Phila Pa 1976) 2017;42:217-23.

10. Magerl FP. Stabilization of the lower thoracic and lumbar spine with external skeletal fixation. Clin Orthop Relat Res 1984;189:125-41.

11. Kabins MB, Weinstein JN. The History of Vertebral Screw and Pedicle Screw Fixation. Iowa Orthop J 1991;11:127-36.

12. Foley KT, Gupta SK. Percutaneous pedicle screw fixation of the lumbar spine: Preliminary clinical results. J Neurosurg 2002;97:7-12.

13. Lowery GL, Kulkarni SS. Posterior percutaneous spine instrumentation. Eur Spine J 2000;9 Suppl 1:S126-30.

14. Vaccaro AR, Rizzolo SJ, Balderston RA, et al. Placement of pedicle screws in the thoracic spine: Part II: An anatomical and radiographic assessment. J Bone Joint Surg Am 1995;77:1200-6.

15. Leu H, Hauser R, Schreiber A. Percutaneous lumbar spine fusion. Acta Orthop Scand Suppl 1993;251:116-9.

16. Wiesner L, Kothe R, Rüther W. Anatomic Evaluation of Two Different Techniques for the Percutaneous Insertion of Pedicle Screws in the Lumbar Spine. Spine (Phila Pa 1976) 1999;24:1599-603.

17. Tabaraee E, Gibson AG, Karahalios DG, et al. Intraoperative Cone Beam-Computed Tomography With Navigation (O-ARM) Versus Conventional Fluoroscopy (C-ARM). Spine (Phila Pa 1976) 2013;38:1953-8.

18. Abdullah KG, Bishop FS, Lubelski D, et al. Radiation exposure to the spine surgeon in lumbar and thoracolumbar fusions with the use of an intraoperative computed tomographic 3-dimensional imaging system. 
Spine (Phila Pa 1976) 2012;37:E1074-8.

19. Bratschitsch G, Leitner L, Stücklschweiger G, et al. Radiation Exposure of Patient and Operating Room Personnel by Fluoroscopy and Navigation during Spinal Surgery. Sci Rep 2019;9:17652.

20. Tian NF, Wu Y Sen, Zhang XL, et al. Minimally invasive versus open transforaminal lumbar interbody fusion: A meta-analysis based on the current evidence. Eur Spine J 2013;22:1741-9.

21. Klingler JH, Sircar R, Scheiwe C, et al. Comparative Study of C-arms for Intraoperative 3-dimensional Imaging and Navigation in Minimally Invasive Spine Surgery Part I: Applicability and Image Quality. Clin Spine Surg 2017;30:276-84.

22. Wang MY, Kim KA, Liu CY, et al. Reliability of threedimensional fluoroscopy for detecting pedicle screw violations in the thoracic and lumbar spine. Neurosurgery 2004;54:1138-42; discussion 1142-3.

23. Kosmopoulos V, Schizas C. Pedicle screw placement accuracy: A meta-analysis. Spine (Phila Pa 1976) 2007;32:E111-20.

24. Mason A, Paulsen R, Babuska JM, et al. The accuracy of pedicle screw placement using intraoperative image guidance systems: A systematic review. Spine J 2017;17:1215-29.

25. Nelson EM, Monazzam SM, Kim KD, et al. Intraoperative fluoroscopy, portable X-ray, and CT: Patient and operating room personnel radiation exposure in spinal surgery. Spine J 2014;14:2985-91.

26. Villard J, Ryang YM, Demetriades AK, et al. Radiation exposure to the surgeon and the patient during posterior lumbar spinal instrumentation: A prospective randomized comparison of navigated versus non-navigated freehand techniques. Spine (Phila Pa 1976) 2014;39:1004-9.

27. Mendelsohn D, Strelzow J, Dea N, et al. Patient and surgeon radiation exposure during spinal instrumentation using intraoperative computed tomography-based navigation. Spine J 2016;16:343-54.

28. Dabaghi Richerand A, Christodoulou E, Li Y, et al. Comparison of effective dose of radiation during pedicle screw placement using intraoperative computed tomography navigation versus fluoroscopy in children with spinal deformities. J Pediatr Orthop 2016;36:530-3.

29. Riis J, Lehman RR, Perera RA, et al. A retrospective comparison of intraoperative CT and fluoroscopy evaluating radiation exposure in posterior spinal fusions for scoliosis. Patient Saf Surg 2017;11:32.

30. Mezger U, Jendrewski C, Bartels M. Navigation in surgery. Langenbecks Arch Surg 2013;398:501-14.

31. Brodwater BK, Roberts DW, Nakajima T, et al. Extracranial application of the frameless stereotactic operating microscope: Experience with lumbar spine. Neurosurgery 1993;32:209-213.

32. Holly LT, Foley KT. Intraoperative spinal navigation. Spine (Phila Pa 1976) 2003;28:S54-61.

33. Gelalis ID, Paschos NK, Pakos EE, et al. Accuracy of pedicle screw placement: a systematic review of prospective in vivo studies comparing free hand, fluoroscopy guidance and navigation techniques. Eur Spine J 2012;21:247-55.

34. Girardi FP, Cammisa FP, Sandhu HS, et al. The placement of lumbar pedicle screws using computerised stereotactic guidance. J Bone Joint Surg Br 1999;81:825-9.

35. Amiot LP, Lang K, Putzier M, et al. Comparative results between conventional and computer-assisted pedicle screw installation in the thoracic, lumbar, and sacral spine. Spine (Phila Pa 1976) 2000;25:606-14.

36. Shin BJ, James AR, Njoku IU, et al. Pedicle screw navigation: A systematic review and meta-analysis of perforation risk for computer-navigated versus freehand insertion - A review. J Neurosurg Spine 2012;17:113-22.

37. Smith ZA, Sugimoto K, Lawton CD, et al. Incidence of lumbar spine pedicle breach after percutaneous screw fixation: a radiographic evaluation of 601 screws in 151 patients. J Spinal Disord Tech 2014;27:358-63.

38. Parker SL, McGirt MJ, Farber SH, et al. Accuracy of free-hand pedicle screws in the thoracic and lumbar spine: analysis of 6816 consecutive screws. Neurosurgery 2011;68:170-8.

39. Sembrano JN, Polly DW Jr, Ledonio CG, et al. Intraoperative 3-dimensional imaging (O-arm) for assessment of pedicle screw position: Does it prevent unacceptable screw placement? Int J Spine Surg 2012;6:49-54.

40. Van de Kelft E, Costa F, Van der Planken D, et al. A prospective multicenter registry on the accuracy of pedicle screw placement in the thoracic, lumbar, and sacral levels with the use of the $\mathrm{O}$-arm imaging system and StealthStation Navigation. Spine (Phila Pa 1976) 2012;37:E1580-7.

41. Dusad T, Kundnani V, Dutta S, et al. Comparative prospective study reporting intraoperative parameters, pedicle screw perforation, and radiation exposure in navigation-guided versus non-navigated fluoroscopyassisted minimal invasive transforaminal lumbar interbody fusion. Asian Spine J 2018;12:309-16.

42. Cho $\mathrm{W}$, Cho SK, Wu C. The biomechanics of pedicle 
screw-based instrumentation. J Bone Joint Surg Br 2010;92:1061-5.

43. Kim CW, Lee YP, Taylor W, et al. Use of navigationassisted fluoroscopy to decrease radiation exposure during minimally invasive spine surgery. Spine J 2008;8:584-90.

44. Hubbe U, Sircar R, Scheiwe C, et al. Surgeon, staff, and patient radiation exposure in minimally invasive transforaminal lumbar interbody fusion: impact of 3D fluoroscopy-based navigation partially replacing conventional fluoroscopy: study protocol for a randomized controlled trial. Trials 2015;16:142.

45. Scheufler KM, Franke J, Eckardt A, et al. Accuracy of image-guided pedicle screw placement using intraoperative computed tomography-based navigation with automated referencing. Part II: thoracolumbar spine. Neurosurgery 2011;69:1307-16.

46. Nathoo N, Çavuşoğlu MC, Vogelbaum MA, et al. In touch with robotics: Neurosurgery for the future. Neurosurgery 2005;56:421-33.

47. Ahern DP, Gibbons D, Schroeder GD, et al. Imageguidance, Robotics, and the Future of Spine Surgery. Clin Spine Surg 2020;33:179-84.

48. Schroerlucke SR, Wang MY, Cannestra AF, et al. Complication Rate in Robotic-Guided vs Fluoro-Guided Minimally Invasive Spinal Fusion Surgery: Report from MIS Refresh Prospective Comparative Study. Spine J 2017;17:S254-5.

49. Kantelhardt SR, Martinez R, Baerwinkel S, et al.

Cite this article as: Weiner JA, McCarthy MH, Swiatek P, Louie PK, Qureshi SA. Narrative review of intraoperative image guidance for transforaminal lumbar interbody fusion. Ann Transl Med 2021;9(1):89. doi: 10.21037/atm-20-1971
Perioperative course and accuracy of screw positioning in conventional, open robotic-guided and percutaneous robotic-guided, pedicle screw placement. Eur Spine J 2011;20:860-8.

50. Overley SC, Cho SK, Mehta AI, et al. Navigation and robotics in spinal surgery: Where are we now? Neurosurgery 2017;80:S86-99.

51. Kim HJ, Kang KT, Park SC, et al. Biomechanical advantages of robot-assisted pedicle screw fixation in posterior lumbar interbody fusion compared with freehand technique in a prospective randomized controlled trialperspective for patient-specific finite element analysis. Spine J 2017;17:671-80.

52. Snyder LA. Integrating robotics into a minimally invasive transforaminal interbody fusion workflow. Neurosurg Focus 2018;45:V4.

53. Chenin L, Peltier J, Lefranc M. Minimally invasive transforaminal lumbar interbody fusion with the ROSATM Spine robot and intraoperative flat-panel CT guidance. Acta Neurochir (Wien) 2016;158:1125-8.

54. Du JP, Fan Y, Liu JJ, et al. Application of Gelatin Sponge Impregnated with a Mixture of 3 Drugs to Intraoperative Nerve Root Block Combined with Robot-Assisted Minimally Invasive Transforaminal Lumbar Interbody Fusion Surgery in the Treatment of Adult Degenerative Scoliosis: A Clinical Observation Including 96 Patients. World Neurosurg 2017;108:791-7. 